\title{
Fractional differintegral transformations of univalent Meijer's G-functions
}

\author{
Amir Pishkoo and Maslina Darus ${ }^{*}$
}

\footnotetext{
* Correspondence: maslina@ukm. my

School of Mathematical Sciences, Faculty of Science and Technology, Universiti Kebangsaan Malaysia, Bangi 43600, Selangor Darul Ehsan, Malaysia
}

\begin{abstract}
In this article, the univalent Meijer's G-functions are classified into three types. Certain integral, differential or differintegral transformations preserving the univalence of the Meijer's G-functions, have been discussed. This classification and transformations are based on Kiryakova's studies in representing the generalized hypergeometric functions as fractional differintegral operators of three basic elementary functions. In fact, these transformations are the Erdélyi-Kober operators $(m=1)$ or their two-tuple compositions (for $m=2$ ) known also as hypergeometric fractional differintegrals. A number of new univalent Meijer's G-functions can be obtained by successive applications of such transformations, being operators of the generalized fractional calculus (GFC). Some new relations are then interpreted for the starlike, convex, and positive real part functions in terms of Meijer's G-functions.
\end{abstract}

Mathematics Subject Classification (2000): 30C45; 33C60; 33E20.

Keywords: Meijer's G-function, univalent functions, generalized fractional calculus, Erdélyi-Kober operators, starlike functions, convex functions, positive real part functions

\section{Introduction}

One of the main topics in univalent functions theory is dealing with integral or differential operators that are used to obtain new subclasses of univalent functions and their properties. The starting point in this theory is to perceive some transformations, or operators, in which the property of univalence is preserved [1]. These well-known transformations include rotation, dilation and others. To study their action, usually the series representation of the functions in the class $A$ is used. Recently, a very general class of such operators have been defined by means of single integrals (or differintegrals) involving Meijer's G-functions as kernels, the so-called operators of the generalized fractional calculus $(G F C)$, [2]. In [3], Kiryakova et al. proposed sufficient conditions that guarantee the mappings related to these operators to preserve the univalency of the functions. In addition, in [4] they considered also some other mapping, distortion, and characterization properties of the generalized fractional calculus operators involving Meijer's G-functions.

In the recent decades, Meijer's G-function has found various applications in different areas close to applied mathematics such as mathematical physics (hydrodynamics, theory of elasticity, potential theory, etc), theoretical physics, mathematical statistics, queuing theory, optimization theory, sinusoidal signals, generalized birth and death 
processes and many others. Due to the elegant and general properties of the $G$-functions, it has become possible to represent the solutions of many problems in these fields in their terms. Stated in this way, the problems gain a much more general character, due to the great freedom of choice of the orders $m ; n ; p ; q$ and the parameters of the $G$-functions, in comparison to the other special functions. Simultaneously, the calculations become simpler and more unified. An evidence showing the importance of the $G$-functions is given by the fact that the basic elementary functions and most of the special functions of mathematical physics, including the generalized hypergeometric functions, follow as its particular cases. Therefore, each result concerning a G-function has become a key leading to numerous particular results for the Bessel functions, confluent hypergeometric functions, classical orthogonal polynomials, etc, see [2].

It is believed that Meijer's G-functions could be a convenient tool to unify certain works on univalent functions theory; in other words, the results on univalent functions and also on the subclasses of the univalent functions can be represented in the language of Meijer's $G$-functions, denoted by $G_{p, q}^{m, n}$. To work with univalent Meijer's $G$-functions, we need to know some properties of the GFC operators, related to them, and especially their mapping properties. Fortunately, Kiryakova et al. [2,3] provided all the needs to achieve the goals set in the current study. However, the proposed approach is a little bit different and thus, it will be interesting to see that difference.

The content of this article is divided into three main sections: In the first section, the definition of the Meijer's $G$-function, two important properties of Meijer's $G$-functions including a generalized (multiple, $m$-tuple) Erdélyi-Kober $(E-K)$ operator of the integration of fractional multi-order and the corresponding multiple ( $m$-tuple) fractional derivatives of multi-order, are recalled. The second section is devoted to a main lemma related to the transformations of univalent Meijer's $G$-functions. In this section, the authors work with differintegral operators to transform one univalent Meijer's $G$ function of the lower rank to another univalent Meijer's G-function of the upper rank. In fact, these operators originated from the generalized fractional calculus developed by Kiryakova [2]. Fortunately, these transformations can be repeated many times and finally, there will be a lot of univalent Meijer's G-functions, and operators related to them. Indeed, these are the most general transformations that preserve the property of univalence, and this fact gives us a lot of univalent Meijer's $G$-functions by the iteration method. The third section classifies in tables the actions on the G-functions of the operators of GFC for $m=1$ and $m=2$ and illustrates the same for many known operators in the theory of univalent functions. The last section discusses some relationships for the starlike functions, convex functions, and positive real part functions, in the language of Meijers's G-functions.

Definition 1.1. A definition of the Meijer's $G$-function is given by the following path integral in the complex plane, called Mellin-Barnes type integral [2,5-8]:

$$
G_{p, q}^{m, n}\left(\begin{array}{l}
a_{1}, \ldots, a_{p} \\
b_{1}, \ldots, b_{q}
\end{array} \mid z\right)=\frac{1}{2 \pi i} \int_{L} \frac{\prod_{j=1}^{m} \Gamma\left(b_{j}-s\right) \prod_{j=1}^{n} \Gamma\left(1-a_{j}+s\right)}{\prod_{j=m+1}^{q} \Gamma\left(1-b_{j}+s\right) \prod_{j=n+1}^{p} \Gamma\left(a_{j}-s\right)} z^{s} d s .
$$

Here, an empty product means unity and the integers $m ; n ; p ; q$ are called orders of the $G$-function, or the components of the order $(m ; n ; p ; q) ; a_{p}$ and $b_{q}$ are called 
"parameters" and in general, they are complex numbers. The definition holds under the following assumptions: $0 \leq m \leq q$ and $0 \leq n \leq p$, where $m, n, p$, and $q$ are integer numbers. $a_{j}-b_{k} \neq 1,2,3, \ldots$ for $k=1, \ldots, n$ and $j=1,2, \ldots, m$ imply that no pole of any $\Gamma\left(b_{j}-s\right), j=1, \ldots, m$ coincides with any pole of any $\Gamma\left(1-a_{k}+s\right), k=1, \ldots, n$.

Based on the definition, the following basic properties are easily derived:

$$
z^{\alpha} G_{p, q}^{m, n}\left(\begin{array}{l}
\mathbf{a}_{\mathbf{p}} \\
\mathbf{b}_{\mathbf{q}}
\end{array} \mid z\right)=G_{p, q}^{m, n}\left(\begin{array}{l}
\mathbf{a}_{\mathbf{p}}+\alpha \\
\mathbf{b}_{\mathbf{q}}+\alpha
\end{array} \mid z\right)
$$

where the multiplying term $z^{\alpha}$ changes the parameters of the $G$-function; and the derivatives of arbitrary order $k$ can change the $G$-function's orders and parameters:

$$
z^{k} \frac{d^{k}}{d z^{k}} G_{p, q}^{m, n}\left(\begin{array}{l}
\mathbf{a}_{\mathbf{p}} \\
\mathbf{b}_{\mathbf{q}}
\end{array} \mid z\right)=G_{p+1, q+1}^{m, n+1}\left(\begin{array}{l}
0, \mathbf{a}_{\mathbf{p}} \\
\mathbf{b}_{\mathbf{q}}, \mathbf{k}
\end{array} \mid z\right) .
$$

Definition 1.2. (see, Kiryakova [2,9]). Let $m \geq 1$ be integer, $\beta>0, \gamma_{1}, \ldots, \gamma_{m}$ and $\delta_{1} \geq$ $0, \ldots, \delta_{m} \geq 0$ be arbitrary real numbers. By a generalized (multiple, $m$-tuple) $E$ - $K$ operator of the integration of multi-order $\delta=\left(\delta_{1}, \ldots, \delta_{m}\right)$ we mean an integral operator

$$
I_{\beta, m}^{\left(\gamma_{k}\right),\left(\delta_{k}\right)} f(z)=\int_{0}^{1} G_{m, m}^{m, 0}\left[\sigma \mid \begin{array}{c}
\left(\gamma_{k}+\delta_{k}\right)_{1}^{m} \\
\left(\delta_{k}\right)_{1}^{m}
\end{array}\right] f\left(z \sigma^{\frac{1}{\beta}}\right) d \sigma .
$$

Then, each operator of the form

$$
R f(z)=z^{\beta \delta_{0}} I_{\beta, m}^{\left(\gamma_{k}\right),\left(\delta_{k}\right)} f(z)
$$

with the arbitrary $\delta_{0} \geq 0$ is said to be a generalized ( $m$-tuple) operator of the fractional integration of the R-L type, or briefly, a generalized R-L fractional integral.

For $m=1$, arbitrary $\beta>0, \gamma$ and $\delta>0$, the generalized fractional integrals (1.4) coincide with the well-known $E-K$ operators (integrals) from Sneddon [10]; see also Samko et al. [11], Kiryakova [2]:

$$
I_{\beta}^{\gamma, \delta} f(z)=\int_{0}^{1} \frac{(1-\sigma)^{\delta-1} \sigma^{\gamma}}{\Gamma(\delta)} f\left(z \sigma \frac{1}{\beta}\right) d \sigma=I_{\beta, 1}^{\gamma, \delta} f(z) .
$$

Definition 1.3 (see, Kiryakova [2,9]). With the same parameters as in Definition 1.2 and integers $\eta_{k}=\delta_{k}$, if $\delta_{k}$ is integer and $\left[\delta_{k}\right]+1$, if $\delta_{k}$ is non-integer, $k=1, \ldots, m$, the auxiliary differential operator is introduced:

$$
D_{\eta}=\prod_{r=1}^{m} \prod_{j=1}^{\eta_{r}}\left(\frac{1}{\beta} z \frac{d}{d z}+\gamma_{r}+j\right) .
$$

Then, the multiple ( $m$-tuple) $E$ - $K$ fractional derivatives of multi-order $\delta=\left(\delta_{1} \geq 0, \ldots\right.$, $\left.\delta_{m} \geq 0\right)$ are defined by means of the differintegral operators:

$$
D_{\beta, m}^{\left(\gamma_{k}\right),\left(\delta_{k}\right)}=D_{\eta} I_{\beta, m}^{\left(\gamma_{k}+\delta_{k}\right),\left(\eta_{k}-\delta_{k}\right)}=\left[\prod_{r=1}^{m} \prod_{j=1}^{\eta_{r}}\left(\frac{1}{\beta} z \frac{d}{d z}+\gamma_{r}+j\right)\right] I_{\beta, m}^{\left(\gamma_{k}+\delta_{k}\right),\left(\eta_{k}+\delta_{k}\right)},
$$


and the operators of the form

$$
D f(z)=D_{\beta, m}^{\left(\gamma_{k}\right),\left(\delta_{k}\right)} z^{-\delta_{0}} f(z)=z^{-\delta_{0}} D_{\beta, m}^{\left(\gamma_{k}-\left(\frac{\delta_{0}}{\beta}\right)\right),\left(\delta_{k}\right)} f(z),
$$

with $\delta_{0} \geq 0$, are generally called the generalized (multiple, $m$-tuple) fractional derivatives. The generalized fractional derivatives (1.7) and (1.8) are the counterparts of the generalized fractional integrals (1.4) and (1.5).

Definition 1.4. Let $A$ denotes the class of functions of the form [1]:

$$
f(z)=z+\sum_{n=2}^{\infty} a_{n} z^{n}
$$

which are analytic in the unit disk, $U=\left\{\Delta_{1}:|z|<1\right\}$. By $S$, it is denoted the subclass of the univalent functions in $A$ and by $S^{*}$ and $K$-the subclasses of $S$ whose members are starlike (with respect to the origin) and convex in $U$, respectively.

In order to obtain our results, we need the following theorems due to Kiryakova [2].

Theorem 1.1 (see, Kiryakova [2]). Denote by $\mathfrak{H}_{\mu}(\Omega)$, the class of functions having the form $f(z)=z^{\mu} \tilde{f}(z)$, with $\mu \geq 0$ and $\tilde{f}(z)$ analytic in a domain $\Omega$ starlike with respect to $z=0$. Let the conditions

$$
\gamma_{k}>-\frac{\mu}{\beta}-1, \delta_{k}>0, k=1, \ldots, m
$$

be satisfied. Then, the multiple Erdélyi-Kober operator $I_{(\beta), m}^{\left(\gamma_{k}\right),\left(\delta_{k}\right)}$ defined by (1.4) maps the class $\mathfrak{H}_{\mu}(\Omega)$ into itself, preserving the power functions up to a constant multiplier:

$$
I_{(\beta), m}^{\left(\gamma_{k}\right),\left(\delta_{k}\right)} z^{p}=c_{p} z^{p}, p \geq \mu,
$$

$$
\text { with } c_{p}=\prod_{k=1}^{m} \frac{\Gamma\left(\gamma_{k}+\frac{p}{\beta}+1\right)}{\Gamma\left(\gamma_{k}+\delta_{k}+\frac{p}{\beta}+1\right)} .
$$

Hence, the image of the power series $f(z)=z^{\mu} \sum_{n=0}^{\infty} a_{n} z^{n} \in \mathcal{H}_{\mu}\left(\Delta_{R}\right)$ is given by the series

$$
I_{(\beta), m}^{\left(\gamma_{k}\right),\left(\delta_{k}\right)} f(z)=z^{\mu} \sum_{n=0}^{\infty} a_{n} \prod_{k=1}^{m} \frac{\Gamma\left(\gamma_{k}+\frac{n+\mu}{\beta}+1\right)}{\Gamma\left(\gamma_{k}+\delta_{k}+\frac{n+\mu}{\beta} 1\right)} z^{n}
$$

having the same radius of convergence $R>0$ and the same signs of the coefficients. In particular, let the following conditions:

$$
\Omega=U, R=1, \delta_{k}>0, \mu=1, \gamma_{k}>-2, \beta=1 \quad \text { for } \quad k=1, \ldots, m, \quad \mathfrak{H}_{1}=A ，
$$

then, the above general results have as consequences the properties of the multiple Erdélyi-Kober operators in the class $A$. Namely, under these conditions the suitably normed operator (see, [3]) 


$$
I_{1, m}^{\left(\gamma_{k}\right),\left(\delta_{k}\right)} f(z)=z \sum_{n=0}^{\infty} a_{n} \prod_{k=1}^{m} \frac{\Gamma\left(\gamma_{k}+n+2\right)}{\Gamma\left(\gamma_{k}+\delta_{k}+n+2\right)} z^{n}
$$

maps the class $A$ into itself.

Theorem 1.2 (see, Kiryakova [2], composition/decomposition theorem). Under the conditions (1.14), the classical Erdélyi-Kober operators of the form (1.15), $I_{1}^{\gamma_{k}, \delta_{k}}, k=1, \ldots, m$, commute in $A$ and their product

$$
\begin{aligned}
& I_{1}^{\gamma_{m}, \delta_{m}} I_{1}^{\gamma_{m-1}, \delta_{m-1}} \ldots\left(I_{1}^{\gamma_{1}, \delta_{1}} f(z)\right)=\left[\prod_{k=1}^{m} I_{1}^{\gamma_{k}, \delta_{k}}\right] f(z) \\
& \quad=\int_{0}^{1} \underbrace{\ldots}_{m} \int_{0}^{1}\left[\prod_{k=1}^{m} \frac{\left(1-\sigma_{k}\right)^{\delta_{k}-1} \sigma_{k}^{\gamma_{k}}}{\Gamma\left(\delta_{k}\right)}\right] f\left(z \sigma_{1} \ldots \sigma_{m}\right) d \sigma_{1} \ldots d \sigma_{n}
\end{aligned}
$$

can be represented as an $m$-tuple $E$ - $K$ operator (1.4), i.e., by means of a single integral involving a $G$-function:

$$
\left[\prod_{k=1}^{m} I_{1}^{\gamma_{k}, \delta_{k}}\right] f(z)=I_{1, m}^{\left(\gamma_{k}\right),\left(\delta_{k}\right)} f(z)=\int_{0}^{1} G_{m, m}^{m, 0}\left[\sigma \mid \begin{array}{l}
\left(\gamma_{k}+\delta_{k}, 1\right)_{1}^{m} \\
\left(\gamma_{k}, 1\right)_{1}^{m}
\end{array}\right] f(z \sigma) d \sigma, \quad f \in A,
$$

and conversely, under the same conditions, each multiple $E-K$ operator of form (1.4) can be represented as a product (1.16).

\section{Preliminaries}

Proposition 2.1 (Kiryakova [9,12]). All the generalized hypergeometric functions ${ }_{p} F_{q}$ can be considered as generalized (q-tuple) fractional differintegrals (1.4), (1.5), (1.8), and (1.9) of one of the elementary functions:

$$
\left.\cos _{q-p+1}(x)(\text { if } p<q), x^{\alpha} e^{x} \text { (if } p=q\right), x^{\alpha}(1-x)^{\beta}(\text { if } p=q+1) .
$$

Lemma $2.1[2,9,13]$. Let $|z|<\infty(|z|<1$ for $p=q+1)$, then

$$
\begin{aligned}
& {\left[\Gamma\left(a_{p}\right) / \Gamma\left(b_{q}\right)\right]_{p} F_{q}\left(a_{1}, \ldots, a_{p} ; b_{1}, \ldots, b_{q} ; z\right)=} \\
& \left\{\begin{array}{l}
I_{1,1}^{a_{p}-1, b_{q}-a_{p}}\left\{{ }_{p-1} F_{q-1}\left(a_{1}, \ldots, a_{p-1} ; b_{1}, \ldots, b_{q-1} ; z\right)\right\} \text { if } b_{q}>a_{p}, \\
D_{1,1}^{b_{q}-1, a_{p}-b_{q}}\left\{{ }_{p-1} F_{q-1}\left(a_{1}, \ldots, a_{p-1} ; b_{1}, \ldots b_{q-1} ; z\right)\right\} \text { if } b_{q}<a_{p} .
\end{array}\right.
\end{aligned}
$$

The generalized hypergeometric functions ${ }_{p} F_{q}(z)$ are special cases of the Meijer's $G$-functions (see, $[2,9,14]$ ):

$$
{ }_{p} F_{q}\left(a_{1}, \ldots, a_{p} ; b_{1}, \ldots, b_{q} ; z\right)=\frac{\prod_{j=1}^{q} \Gamma\left(b_{j}\right)}{\prod_{j=1}^{p} \Gamma\left(a_{j}\right)} G_{p, q+1}^{1, p}\left[\begin{array}{l}
1-a_{1}, \ldots, 1-a_{p} \\
0,1-b_{1}, \ldots, 1-b_{q}
\end{array} \mid-z\right],
$$

and this plays an important role in obtaining our results in the current study. The representation (2.2) can be written in the following form for the three cases: (a) $p=0$, $q=1$; (b) $p=1, q=1$; (c) $p=1, q=0$ :

$$
(a){ }_{0} F_{1}=\Gamma\left(b_{1}\right) G_{0,2}^{1,0}\left[\begin{array}{c}
- \\
0,1-b_{1}
\end{array} \mid z\right] ;(b){ }_{1} F_{1}=\frac{\Gamma\left(b_{1}\right)}{\Gamma\left(a_{1}\right)} G_{1,2}^{1,1}\left[\begin{array}{c}
1-a_{1} \\
0,1-b_{1}
\end{array} \mid-z\right] ;(c){ }_{1} F_{0}=\frac{1}{\Gamma\left(a_{1}\right)} G_{1,1}^{1,1}\left[\begin{array}{l}
1-a_{1} \\
0
\end{array} \mid-z\right] .
$$


Thus, it happens to be sufficient that we consider the three basic univalent Meijer's $G$-functions; $G_{0,2}^{1,0} ; G_{1,2}^{1,1} ; G_{1,1}^{1,1}$ and then, a lot of univalent Meijer's $G$-functions can be obtained by using the following approach based on [9]:

Proposition 2.2. All of the univalent Meijer's $G$-functions, $G_{p, q+1}^{1, p}$, can be considered as the generalized ( $q$-tuple) fractional differintegrals (1.4), (1.5), (1.8), and (1.9) of one of the three simplest univalent $G$-functions, namely, $G_{0,2}^{1,0}, G_{1,2}^{1,1}$, and $G_{1,1}^{1,1}$, depending on whether $p<q, p=q, p=q+1$.

Lemma 2.1 can also be easily rewritten in the context of the present study, in terms of the $G$-functions:

Lemma 2.2. Let $|z|<\infty(|z|<1$ for $p=q+1)$, then

$$
\begin{aligned}
& G_{p, q+1}^{1, p}\left(\begin{array}{l}
\left.1-a_{1}, \ldots, 1-a_{p} \mid-z\right) \\
0,1-b_{1}, \ldots, 1-b_{q}
\end{array} \mid-z\right) \\
& =\left\{\begin{array}{c}
I_{1,1}^{a_{p}-1, b_{q}-a_{p}}\left\{G_{p-1, q}^{1, p-1}\left(\begin{array}{c}
1-a_{1}, \ldots, 1-a_{p-1} \\
0,1-b_{1}, \ldots, 1-b_{q-1}
\end{array} \mid-z\right)\right\} \quad \text { if } \quad b_{q}>a_{p}, \\
D_{1,1}^{b_{q}-1, a_{p}-b_{q}}\left\{G_{p-1, q}^{1, p-1}\left(\begin{array}{c}
1-a_{1}, \ldots, 1-a_{p-1} \\
0,1-b_{1}, \ldots, 1-b_{q-1}
\end{array} \mid-z\right)\right\} \quad \text { if } \quad b_{q}<q_{p} .
\end{array}\right.
\end{aligned}
$$

\section{Classification tables for the actions of the single and double $E-K$ operators}

It is believed that Lemma 2.2 is the best inspiration for the current research here, to provide some operators that can transform a Meijer's $G$-function " $G_{p, q}^{m, n_{u}}$ into another

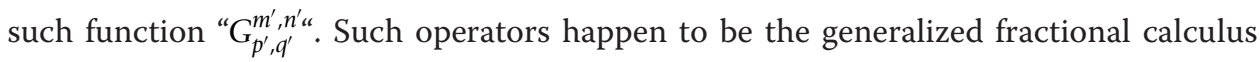
operators by Kiryakova [2].

The univalent Meijer's $G$-functions are classified into three types (depending on the relationship between orders $p$ and $q$, Proposition 2.2) and due to Lemma 2.2, a number of transformations between two different $G$-functions can be summarized and classified as in Tables 1, 2, 3, and 4 .

In Table 1, a classification is made for the operators that transform the three basic Meijer's $G$-functions $\left(G_{1,1}^{1,1}, G_{1,2}^{1,1}, G_{0,2}^{1,0}\right)$ into other $G$-functions, depending on the three cases $p=q+1, p=q$ and $p<q$. Here, we consider single (classical) simple ErdélyiKober integrals (1.6) or the respective $E$ - $K$ derivatives.

Recently Kiryakova et al. [2,3] obtained ones of the most general fractional differintegral operators, generalizing many well-known operators in the univalent function theory. In Table 2, some of these known operators are shown to transform the three basic classes of Meijer's G-functions. An important point here is that instead of presenting them in the form of $E-K$ operators (1.6), we find it better to work in the terms used in Table 2, then we could try to study these operators.

In Table 3, we show the action of compositions of two $E-K$ operators (in sense of Theorem 1.2), the so-called two-tuple $E-K$ operators (they can be two-tuple "integral" operators, two-tuple "differential" operators or the two-tuple mixed "differintegral" operators) to transform $G_{p, q+1}^{1, p}$-functions with $p=q+1$. Let us note that these operators appear as special cases of Definitions 1.2 and 1.3 when $m=2$, and are also called "hypergeometric fractional integrals and derivatives". The conditions on the parameters $a_{k+1}$ and $b_{k}, k=1,2,3$ in the column "conditions" determine the form and the kind of the operators. 
Table 1 The effect of the Erdélyi-Kober operators on the Meijer's $G_{p, q+1}^{1, p}$-functions

\begin{tabular}{lll}
\hline Operators & $(p=q+1=1)$ & $(p=q=1)$ \\
\hline$I_{1,1}^{a_{2}-1, b_{1}-a_{2}}$ & $G_{1,1}^{1,1} \rightarrow G_{2,2}^{1,2}\left[\begin{array}{l}1-a_{1}, 1-a_{2} \\
0,1-b_{1}\end{array}\right)$ & \\
$D_{1,1}^{b_{1}-1, a_{2}-b_{1}}$ & $G_{1,1}^{1,1} \rightarrow\left(b_{1,2}>a_{2,2}\left(b_{1}\right)<a_{2}\right)$ & \\
$I_{1,1}^{a_{2}-1, b_{2}-a_{2}}\left(b_{2}>a_{2}\right)$ & & $G_{1,2}^{1,1} \rightarrow G_{2,3}^{1,2}\left[\begin{array}{l}1-a_{1}, 1-a_{2} \\
\left.0,1-b_{1}, 1-b_{2} \mid-z\right]\end{array}\right]$ \\
$D_{1,1}^{b_{2}-1, a_{2}-b_{2}}\left(b_{2}<a_{2}\right)$ & $G_{1,2}^{1,1} \rightarrow G_{2,3}^{1,2}$ \\
$I_{1,1}^{a_{1}-1, b_{2}-a_{1}}\left(b_{2}>a_{1}\right)$ & \\
$D_{1,1}^{b_{2}-1, a_{1}-b_{2}}\left(b_{2}<a_{1}\right)$ & & \\
\hline
\end{tabular}


Table 2 The effect of the well-known Erdélyi-Kober operators on Meijer's $G_{p, q+1}^{1, p}$-function

\begin{tabular}{|c|c|}
\hline Operators & Transformation \\
\hline$I_{1,1}^{-1,1}$ (Biernacki) & $\begin{array}{l}G_{1,1}^{1,1} \rightarrow G_{2,2}^{1,2}\left[\begin{array}{l}1-a_{1}, 1 \\
0,0\end{array}-z\right] \\
\left(b_{1}=1, a_{2}=0, b_{1}>a_{2} ; p=1, q=0\right)\end{array}$ \\
\hline $2 I_{1,1}^{0,1}$ (Libera) & $\begin{aligned} G_{1,1}^{1,1} & \rightarrow 2 G_{2,2}^{1,2}\left[\begin{array}{l}1-a_{1}, 0 \\
0,-1\end{array} \mid-z\right] \\
\left(b_{1}\right. & \left.=2, a_{2}=1, b_{1}>a_{2} ; p=1, q=0\right)\end{aligned}$ \\
\hline$\frac{1}{\Gamma(\alpha+1)} D_{1,1}^{-1, \alpha}$ (Ruscheweyh) & $\begin{aligned} G_{1,1}^{1,1} & \rightarrow \frac{1}{\Gamma(\alpha+1)} G_{2,2}^{1,2}\left[\begin{array}{l}1-a_{1}, 1-\alpha \\
0,1\end{array} \mid-z\right] \\
\left(b_{1}\right. & \left.=0, a_{2}=\alpha, ; p=2, q=1\right)\end{aligned}$ \\
\hline$I_{1,1}^{-1,1}$ & $\begin{array}{l}G_{1,2}^{1,1} \rightarrow G_{2,3}^{1,2}\left[\begin{array}{l}\left.1-a_{1,1}, 1-z\right] \\
0,1-b_{1}, 0\end{array}\right] \\
\left(b_{2}=1, a_{2}=0, b_{2}>a_{2} ; p=q=1\right)\end{array}$ \\
\hline $2 I_{1,1}^{0,1}$ & $\begin{aligned} G_{1,2}^{1,1} & \rightarrow 2 G_{2,3}^{1,2}\left[\begin{array}{l}1-a_{1}, 0 \\
0,1-b_{1},-1\end{array} \mid-z\right] \\
\left(b_{2}\right. & \left.=2, a_{2}=1, b_{2}>a_{2} ; p=q=1\right)\end{aligned}$ \\
\hline$\frac{1}{\Gamma\left(x^{1}\right)} D_{1,1}^{-1, \alpha}$ & $G_{1,2}^{1,1} \rightarrow \frac{1}{\Gamma(\alpha+1)} G_{2,3}^{1,2}\left[\begin{array}{l}1-a_{1}, 1-\alpha \\
0,1-b_{1}, 1\end{array} \mid-z\right]$ \\
\hline$\Gamma(\alpha+1)^{1,1}$ & $\left(b_{2}=0, a_{2}=\alpha ; p=q=1\right)$ \\
\hline$I_{1,1}^{-1,1}$ & $\begin{aligned} G_{0,2}^{1,0} & \rightarrow G_{1,3}^{1,1}\left[\begin{array}{l}1 \\
0,1-b_{1}, 0\end{array} \mid-z\right] \\
\left(b_{2}\right. & \left.=1, a_{1}=0, b_{2}>a_{1} ; p=0, q=1\right)\end{aligned}$ \\
\hline $2 I_{1,1}^{0,1}$ & $\begin{aligned} & G_{0,2}^{1,0} \rightarrow 2 G_{1,3}^{1,1}\left[\begin{array}{l}0 \\
0,1-b_{1},-1\end{array} \mid-z\right] \\
& \quad\left(b_{2}-2, a_{1}=1, b_{2}>a_{1} ; p=0, q=1\right)\end{aligned}$ \\
\hline$\frac{1}{\Gamma(\alpha+1)} D_{1,1}^{-1, \alpha}$ & $\begin{aligned} G_{0,2}^{1,0} & \rightarrow \frac{1}{\Gamma(\alpha+1)} G_{1,3}^{1,1}\left[\begin{array}{l}1-\alpha \\
0,1-b_{1}, 1\end{array} \mid-z\right] \\
\left(b_{2}\right. & \left.=0, a_{1}=\alpha ; p=0, q=1\right)\end{aligned}$ \\
\hline
\end{tabular}

In Table 4, the compositions of two classical $E-K$ operators (again the case $m=2$ ) act on the Meijer's $G_{p, q+1}^{1, p}$-function with $p=q=1$.

Finally, in Table 5, the third classified $G_{p, q+1}^{1, p}$-function with $p<q, p=0, q=1$ is shown has transformed by the action of these two-tuple $E$ - $K$ operators, so to obtain new univalent Meijer's G-functions.

Table 3 The effect of the two-tuple Erdélyi-Kober operators on the Meijer's $G_{p, q+1}^{1, p}$-function $(\boldsymbol{p}=\boldsymbol{q}+\mathbf{1}=\mathbf{1})$

\begin{tabular}{lcc}
\hline Operators & Conditions & Transformation \\
\hline$I_{1,1}^{a_{3}-1, b_{2}-a_{3}} I_{1,1}^{a_{2}-1, b_{1}-a_{2}}$ & $b_{1}>a_{2}$ and $b_{2}>a_{3}$ & $G_{1,1}^{1,1} \rightarrow G_{3,3}^{1,3}\left[\begin{array}{l}1-a_{1}, 1-a_{2}, 1-a_{3} \\
0,1-b_{1}, 1-b_{2}\end{array}\right]$ \\
$D_{1,1}^{b_{2}-1, a_{3}-b_{2}} I_{1,1}^{a_{2}-1, b_{1}-a_{2}}$ & $b_{1}>a_{2}$ and $b_{2}<a_{3}$ & $G_{1,1}^{1,1} \rightarrow G_{3,3}^{1,3}$ \\
$I_{1,1}^{a_{3}-1, b_{2}-a_{3}} D_{1,1}^{b_{1}-1, a_{2}-b_{1}}$ & $b_{1}<a_{2}$ and $b_{2}>a_{3}$ & $G_{1,1}^{1,1} \rightarrow G_{3,3}^{1,3}$ \\
$D_{1,1}^{b_{2}-1, a_{3}-b_{2}} D_{1,1}^{b_{1}-1, a_{2}-b_{1}}$ & $b_{1}<a_{2}$ and $b_{2}<a_{3}$ & $G_{1,1}^{1,1} \rightarrow G_{3,3}^{1,3}$
\end{tabular}


Table 4 The effect of the two-tuple Erdélyi-Kober operators on the Meijer's $G_{p, q+1}^{1, p}$-function $(p=q=1)$

\begin{tabular}{lcc}
\hline Operators & Conditions & Transformation \\
\hline$I_{1,1}^{a_{3}-1, b_{3}-a_{3}} I_{1,1}^{a_{2}-1, b_{2}-a_{2}}$ & $b_{2}>a_{2}$ and $b_{3}>a_{3}$ & $\left.G_{1,2}^{1,1} \rightarrow G_{3,4}^{1,3}\left[\begin{array}{l}1-a_{1}, 1-a_{2}, 1-a_{3} \\
0,1-b_{1}, 1-b_{2}, 1-b_{3}\end{array}\right]-z\right]$ \\
$D_{1,1}^{b_{3}-1, a_{3}-b_{3}} I_{1,1}^{a_{2}-1, b_{2}-a_{2}}$ & $b_{2}>a_{2}$ and $b_{3}<a_{3}$ & $G_{1,2}^{1,1} \rightarrow G_{3,4}^{1,3}$ \\
$I_{1,1}^{a_{3}-1, b_{3}-a_{3}} D_{1,1}^{b_{2}-1, a_{2}-b_{2}}$ & $b_{2}<a_{2}$ and $b_{3}>a_{3}$ & $G_{1,2}^{1,1} \rightarrow G_{3,4}^{1,3}$ \\
$D_{1,1}^{b_{3}-1, a_{3}-b_{3}} D_{1,1}^{b_{2}-1, a_{2}-b_{2}}$ & $b_{2}<a_{2}$ and $b_{3}<a_{3}$ & $G_{1,2}^{1,1} \rightarrow G_{3,4}^{1,3}$ \\
\hline
\end{tabular}

4 The starlike, convex, and positive real part G-functions

There is an elementary and beautiful relationship between the convex and starlike functions that was first noticed by Alexander $[1,15]$. The form of this relation can be now rewritten in the language of the Meijer $G$-functions and the fractional differintegral operators, as follows:

$$
\begin{aligned}
& \text { if } G_{p-1, q}^{1, p-1} \text { is a convex function, then } D_{1}^{-1,1} G_{p-1, q}^{1, p-1} \text { is a starlike function. } \\
& \text { if } \mathrm{G}_{p^{\prime}-1, q^{\prime}}^{1, p^{\prime}-1} \text { is a starlike function, then } I_{1}^{-1,1} G_{p^{\prime}-1, q^{\prime}}^{1, p^{\prime}-1} \text { is a convex function. }
\end{aligned}
$$

As a fact, the operators $D_{1}^{-1,1}$, and $I_{1,1}^{-1,1}$ are the $E-K$ operators from Table 1 (or Table 2).

If the Noshiro-Warschawski theorem [1] is used (if $\operatorname{Re}(f(z))>0$ for all $z$ in a convex domain $D$, and $f(z)$ is univalent in $D$ ), a lot of inequality relations can then be obtained for the Meijer G-functions. All the univalent Meijer's G-functions in our Tables 1, 2, 3, and 4 can be used and it is deduced that $\operatorname{Re}\left(G_{1,1}^{\prime 1,1}\right)>0, \operatorname{Re}\left(G_{2,2}^{\prime 1,2}\right)>0, \ldots$, etc. In other words, all the functions $G_{1,1}^{\prime 1,1}, G_{2,2}^{\prime 1,2}, \ldots$, etc. belong to the functions with a positive real part.

\section{Conclusions}

After the classification results in Proposition 2.2 and Lemma 2.2 (as consequences of Kiryakova's works), in this article the univalent Meijer's $G$-functions are studied under the action of the classical $E-K$ operators and their two-tuple compositions. It happens that it is enough to use the differintegral operators of the GFC [2], for $m=1,2$. By means of such approach, based on these operators, and using some statements from the theory of the GFC, some new relationships for the starlike and convex functions and also the functions with positive real part can be interpreted in terms of the Meijer's $G$-functions.

Table 5 The effect of the two-tuple Erdélyi-Kober operators on the Meijer's $G_{p, q+1}^{1, p}$-function ( $\left.p<q, p=0, q=1\right)$

\begin{tabular}{lcc}
\hline Operators & Conditions & Transformation \\
\hline$I_{1,1}^{a_{2}-1, b_{3}-a_{2}} I_{1,1}^{a_{1}-1, b_{2}-a_{1}}$ & $b_{2}>a_{1}$ and $b_{3}>a_{2}$ & $\left.G_{0,2}^{1,0} \rightarrow G_{2,4}^{1,2}\left[\begin{array}{l}1-a_{1}, 1-a_{2} \\
0,1-b_{1}, 1-b_{2}, 1-b_{3}\end{array}\right]-z\right]$ \\
$D_{1,1}^{b_{3}-1, a_{2}-b_{3}} I_{1,1}^{a_{1}-1, b_{2}-a_{1}}$ & $b_{2}>a_{1}$ and $b_{3}<a_{2}$ & $G_{0,2}^{1,0} \rightarrow G_{2,4}^{1,2}$ \\
$I_{1,1}^{a_{2}-1, b_{3}-a_{2}} D_{1,1}^{b_{1}-1, a_{1}-b_{2}}$ & $b_{2}<a_{1}$ and $b_{3}>a_{2}$ & $G_{0,2}^{1,0} \rightarrow G_{2,4}^{1,2}$ \\
$D_{1,1}^{b_{3}-1, a_{2}-b_{3}} D_{1,1}^{b_{1}-1, a_{1}-b_{2}}$ & $b_{2}<a_{1}$ and $b_{3}<a_{2}$ & $G_{0,2}^{1,0} \rightarrow G_{2,4}^{1,2}$ \\
\hline
\end{tabular}




\section{Acknowledgements}

This study was supported by MOHE with the grant number: UKM-ST-06-FRGS0244-2010. The authors also would like to thank the two referees for giving such informative and critical views to improve the contents of this article.

\section{Authors' contributions}

AP is currently a PhD student under supervision of the MD and jointly worked on deriving the results. All authors read and approved the final manuscript.

\section{Competing interests}

The authors declare that they have no competing interests.

Received: 4 July 2011 Accepted: 16 February 2012 Published: 16 February 2012

\section{References}

1. Duren, PL: Univalent Functions. Springer-Verlag, New York (1983)

2. Kiryakova, V: Generalized Fractional Calculus and Applications. Longman, Harlow, UK (1994)

3. Kiryakova, V, Saigo, M, Srivastava, HM: Some criteria for univalence of analytic Functions involving generalized fractional calculus operators. Fract Calc Appl Anal. 1, 79-104 (1998)

4. Kiryakova, V, Saigo, M, Owa, S: Distortion and characterization theorems for starlike and convex functions related to generalized fractional calculus. Res Inst Math Sci, Kyoto Univ, Proc Kyoto Seminar on Unival F-s $25-46$ (1997). March 24271997

5. Andrews, LC: Special Functions for Engineers and Applied Mathematicians. MacMil-lan, New York (1985)

6. Luke, YL: The Special Functions and Their Approximations. Complex Variables, Academic Press, New Yorkl (1969)

7. Klimyik, AU: Meijer G-function. In: Michiel, H (eds.) Encyclopaedia of Mathematics. Springer, Berlin (2001). ISBN 9781556080104

8. Askey, RA: Meijer G-function. In: Adri D, Olde B (eds.) NIST Handbook of Mathematical Functions. Cambridge University Press, Cambridge (2010). ISBN 978-051192255

9. Kiryakova, V: All the special functions are fractional differintegrals of elementary functions. J Phys A: Math Gen. 30 5085-5103 (1997). doi:10.1088/0305-4470/30/14/019

10. Sneddon, I: The use in mathematical analysis of the Erdélyi-Kober operators and some of their applications in fractional calculus and applications. Proc Int Conf (New Haven) (Lecture Notes in Math 457). pp. 37-79.Springer, Berlin (1975)

11. Samko, SG, Kilbas, AA, Marichev, Ol: Fractional Integrals and Derivatives. Gordon and Breach, Yverdon, Switzerland (1993)

12. Kiryakova, V: The special functions of fractional calculus as generalized fractional calculus operators of some basic functions. Comput Math Appl. 59, 1128-1141 (2010). doi:10.1016/j.camwa.2009.05.014

13. Lavoie, JL, Osler, TJ, Tremblay, R: Fractional derivatives and special functions. SIAM Rev. 18, $24-68$ (1976)

14. Erdélyi, A, Magnus, W, Oberhettinger, F, Tricomi, F: Higher Transcendental Functions. McGraw-Hill, Book Company, Inc, New York1-3 (1953)

15. Alexander, JW: Functions that map the interior of the unit circle upon simple regions. Ann of Math. 17, 12-22 (1915). doi: $10.2307 / 2007212$

doi:10.1186/1029-242X-2012-36

Cite this article as: Pishkoo and Darus: Fractional differintegral transformations of univalent Meijer's $\mathbf{G}$-functions. Journal of Inequalities and Applications 2012 2012:36.

\section{Submit your manuscript to a SpringerOpen ${ }^{\ominus}$ journal and benefit from:}

- Convenient online submission

- Rigorous peer review

- Immediate publication on acceptance

- Open access: articles freely available online

- High visibility within the field

- Retaining the copyright to your article

Submit your next manuscript at $\gg$ springeropen.com 